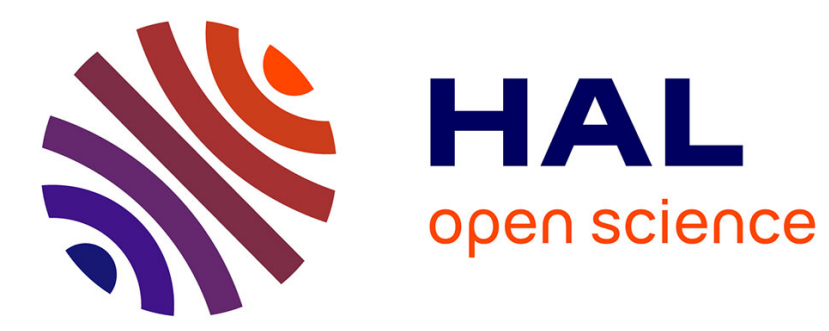

\title{
Benefits and gaps in area-based management tools for the ocean Sustainable Development Goal
}

Julie M Reimer, Rodolphe Devillers, Joachim Claudet

\section{To cite this version:}

Julie M Reimer, Rodolphe Devillers, Joachim Claudet. Benefits and gaps in area-based management tools for the ocean Sustainable Development Goal. Nature Sustainability, 2021, 4, pp.349-357. 10.1038/s41893-020-00659-2 . hal-03206472

\section{HAL Id: hal-03206472 \\ https://hal.science/hal-03206472}

Submitted on 23 Apr 2021

HAL is a multi-disciplinary open access archive for the deposit and dissemination of scientific research documents, whether they are published or not. The documents may come from teaching and research institutions in France or abroad, or from public or private research centers.
L'archive ouverte pluridisciplinaire $\mathbf{H A L}$, est destinée au dépôt et à la diffusion de documents scientifiques de niveau recherche, publiés ou non, émanant des établissements d'enseignement et de recherche français ou étrangers, des laboratoires publics ou privés. 


\section{Benefits and gaps in area-based management tools for the ocean Sustainable Development Goal}

Julie M Reimer*1, Rodolphe Devillers², Joachim Claudet $^{3}$

${ }^{1}$ Department of Geography, Memorial University of Newfoundland, St. John's, Newfoundland, Canada

${ }^{2}$ Espace-Dev (UMR 228), Institut de Recherche pour le Développement (IRD), Maison de la Télédétection, Montpellier, France

${ }^{3}$ National Center for Scientific Research, PSL Université Paris, CRIOBE, USR 3278 CNRS-EPHEUPVD, Maison des Océans, Paris, France

Keywords: Sustainable Development Goal 14, marine spatial planning (MSP), marine protected areas (MPA), area-based management tool (ABMT), other effective area-based conservation measure (OECM), targets, indicators, locally managed marine area (LMMA), territorial user right fishery (TURF), fishery closure

\section{$1 \quad$ ABSTRACT}

2 Sustainable Development Goal (SDG) 14 provides a vision for the world's oceans; however, 3 management interventions needed to achieve SDG 14 remain less clear. We assessed the 4 potential contributions of seven key area-based management tools (such as fisheries closures)

5 to SDG 14 targets. We conducted a rapid systematic review of 177 studies and an expert

6 opinion survey to identify evidence of the ecological, social and economic outcomes from each

7 type of tool. We used these data to assess the level of confidence in the outcomes delivered by

8 each tool and qualitatively scored how each tool contributes to each target. We demonstrate

9 that a combination of tools with diverse objectives and management approaches will be

10 necessary for achieving all SDG 14 targets. We highlight that some tools, including fully and

11 partially protected areas and locally managed marine areas, may make stronger contributions

12 to SDG 14 than others. We identify gaps in the suitability of these tools to some targets, 13 particularly targets related to pollution and acidification, and identify evidence gaps for social 
14 and economic outcomes. Our findings provide operational guidance to support progress toward 15 SDG 14.

17 The United Nations Sustainable Development Goals (SDGs) provide a transformational vision of

18 sustainable development across the environmental, social, and economic dimensions.

19 Sustainable Development Goal 14: Life Below Water (SDG 14) aims to "conserve and

20 sustainably use oceans, seas, and marine resources for sustainable development" ${ }^{1}$. Through its

21 seven primary targets, SDG 14 addresses ocean challenges: reducing marine pollution (SDG

22 14.1), restoring marine ecosystems (SDG 14.2), reducing ocean acidification (SDG 14.3),

23 enabling sustainable fisheries (SDG 14.4), conserving marine areas (SDG 14.5), ending harmful

24 fishery subsidies (SDG 14.6), and increasing economic benefits to Small Island Developing States

25 and least developed countries (SDG 14.7). Achieving SDG 14 was shown to benefit other SDGs,

26 most notably SDG 1: No Poverty and SDG 2: Zero Hunger ${ }^{2,3}$. A focus on sustaining marine

27 ecosystem services may foster fair and equitable benefit-sharing that contributes to multiple

28 interconnected SDGs ${ }^{4}$; however, SDG 14 was criticized for being largely aspirational rather than

29 operational and measurable ${ }^{5}$, and management-level guidance is critically needed to support

30 progress toward SDG 14 targets $^{6-8}$.

Management of human activities and natural resources often has a spatial dimension, reflecting various social, sectoral, or geopolitical boundaries. This spatial lens extends to planning and management of oceans and their natural resources ${ }^{9-12}$. Planning and management can address single-sector needs, like achieving specific fisheries objectives ${ }^{13}$, or multiple objectives in the wider seascape, as with comprehensive ocean zoning or marine spatial planning ${ }^{14,15}$. Both

37 single- and multi-sector spatial plans tend to rely on the implementation of spatial or areabased management tools (ABMTs). ABMTs are geographically defined areas where human activities are regulated for one or more purposes, delivering one or more social and ecological outcomes to achieve objectives for biodiversity conservation, sustainable resource use, or

41 both ${ }^{16,17}$. Since ABMTs are common across ocean sectors, management objectives, and social-

42 ecological contexts ${ }^{10}$, and given their recommended use for achieving conservation and 
43 sustainability goals ${ }^{18,19}$, a clearer understanding of the contributions of ABMTs to SDG 14

44 targets may enable strategic planning toward achieving this goal. Here, we assess evidence of

45 ecological, social, and economic ABMT outcomes, ranging from increasing organism size to 46 maintaining ecosystem resilience to preserving access to resources and cultural traditions. We

47 use this evidence to determine the potential contributions of prominent ABMTs to SDG 14 targets, guiding countries and practitioners in selecting the best interventions to deliver SDG

4914.

50

\section{Assessing evidence of outcomes and contributions to SDG 14}

52 To provide management-level guidance on the use of ABMTs for achieving SDG 14, we

53 conducted a two-step approach, including a rapid systematic review of the scientific literature

54 followed by an expert survey to identify evidence, and gaps in evidence, of 17 ecological, social, 55 and economic outcomes from seven types of ABMTs (Figure 1 and Supplementary Table 1). The

56 selected ABMTs have objectives in biodiversity conservation, sustainable resource use,

57 particularly fisheries, or both (Supplementary Table 2). These ABMTs regulate the activities of

58 single sectors, as is typical of gear restriction areas (GRAs), fishery closures (FCs), territorial user

59 right fisheries (TURFs), and particularly sensitive sea areas (PSSAs), or multiple sectors, as is

60 typical of fully protected areas (FPAs), partially protected areas (PPAs), and locally managed

61 marine areas (LMMAs). Many of these tools may be considered 'other effective area-based

62 conservation measures' (OECMs) in some contexts and these tools may overlap in their

63 application or the types of regulations they use.

Using evidence from the literature and expert opinions, we conducted a confidence assessment of the ability of selected ABMTs to deliver selected outcomes. Confidence level was determined

67 based on "quantity and quality of evidence" and the "level of agreement among evidence",

68 following a similar approach to the Intergovernmental Panel on Biodiversity and Ecosystem

69 Services $^{20}$ (Figure 2, Supplementary Table 6, Supplementary Figure 3). Finally, we developed a

70 scoring system to link ABMTs to SDG 14 targets based on the relative contributions of outcomes

71 to the targets (see Equation 1 in Methods). 


\section{Evidence of ABMT outcomes}

73 We identified 276 records of ABMT outcomes from 177 studies (Table 1 and Supplementary

74 References). Of these studies, 31 reported evidence through synthesis-based literature,

75 including meta-analyses, systematic reviews, or literature reviews. Although we found evidence

76 of fully protected areas (FPAs), partially protected areas (PPAs), gear restriction areas (GRAs),

77 fishery closures (FCs), and territorial user right fisheries (TURFs) in these 31 studies, most

78 reported only ecological outcomes. The majority of records from both synthesis-based evidence

79 and evidence from individual studies reported on outcomes from FPAs and PPAs (25\% and 29\%,

80 respectively). The proportion of all literature-based evidence records across outcomes was

81 comparable for locally managed marine areas (LMMAs), GRAs, FCs, and TURFs (8\%, 14\%, 12\%,

$8212 \%$, respectively); however, no synthesis-based evidence was identified for outcomes of

83 LMMAs. Individual studies reported 204 records of evidence for ABMT outcomes, including 95

84 records of positive effects, 68 records of negative effects, and 41 records documenting no

85 effect of ABMTs on outcomes. No literature-based evidence was identified for outcomes of

86 particularly sensitive sea areas (PSSAs).

Expert opinions collected using survey questionnaires $(n=75)$ exhibited greater agreement on the ecological outcomes of most ABMTs than social and economic outcomes (Figure 3). Experts agreed that for most ABMTs, positive effects would occur for the majority of ecological

91 outcomes. More than $60 \%$ of experts agreed that FPAs, PPAs, LMMAs, and FCs would have 92 positive effects on organism size ( $96 \%, 87 \%, 60 \%$, and $87 \%$, respectively), organism abundance

93 (89\%, 91\%, 67\%, and 89\%, respectively), maintaining habitat (96\%, 92\%, 67\%, and 65\%,

94 respectively), ecosystem function (93\%, 84\%, 63\%, and 61\%, respectively), ecosystem resilience $95(92 \%, 81 \%, 61 \%$, and 63\%, respectively), and on reducing threats to species $(93 \%, 84 \%, 72 \%$, 96 and $87 \%$, respectively). More than $60 \%$ of experts also agreed that GRAs would have positive 97 effects on organism size (68\%), organism abundance (73\%), maintaining habitat (76\%), and 98 reducing threats to species (79\%). 
100 In contrast, at least $25 \%$ of experts agreed that FPAs, PPAs, LMMAs, GRAs, and FCs would have 101 negative effects on harvest earnings (43\%, 25\%, 37\%, and 43\%, respectively), maintaining 102 access to resources (72\%, 53\%, 43\%, and 59\%, respectively), equitable access to resources $103(47 \%, 37 \%, 28 \%$, and 37\%, respectively), and preserving traditions and customs (47\%, 32\%, $10431 \%$, and 40\%, respectively). Experts were less certain about outcomes of TURFs and PSSAs, as 105 more than $20 \%$ of experts indicated either "do not know" or "prefer not to answer" for all 106 outcomes of these tools (see Methods). Several experts commented on the difficulty of 107 providing general information on expected effects of ABMTs, especially those pertaining to 108 social and economic outcomes, given the context-dependency of these outcomes relative to 109 local ABMT objectives, monitoring capacity, and social groups.

\section{Confidence assessment}

112 Based on evidence provided by the literature and by experts, we found generally greater 113 confidence in the delivery of ecological outcomes than social and economic outcomes from the 114 assessed ABMTs (Figure 4). We found high confidence, assigned to "well-established" and 115 "established but incomplete" categories (Figure 2), that multi-sector ABMTs, including FPAs, 116 PPAs, and LMMAs, would have positive effects on organism size and abundance, species 117 diversity, habitat, ecosystem function and resilience, and reducing threats to species. Most 118 single sector ABMTs, including GRAs, FCs, and TURFs, were found to have positive effects on 119 fewer ecological outcomes, including organism size and abundance, ecosystem function, and 120 reducing threats to species, with high confidence. We also found high confidence that the 121 seven ABMTs would have no effect on reducing impacts of acidification. While we found no 122 literature-based evidence (Table 1), there was medium or high agreement among experts that 123 ABMTs would have no effect on this outcome (Figure 3). Similarly, though to a lesser extent, 124 there was agreement that none of the ABMTs studied are likely to reduce pollution, though 125 confidence assessment was "incomplete" for LMMAs and PSSAs. Further, we found that FPAs 126 may have the potential to reduce pollution, though evidence of this outcome was "established

127 but unresolved". Low confidence persisted for all outcomes of PSSAs, except the shared result 128 of no effect of ABMTs on reducing impacts of acidification. 
130 Across ABMTs, low confidence, assigned to "established but unresolved", "unresolved", and

131 "incomplete" categories (Figure 2), was found for 43\% of all assessed outcomes; however,

132 these were assigned to only $25 \%$ of ecological outcomes compared to $63 \%$ of social and

133 economic outcomes. There is an apparent lack of evidence of social and economic outcomes

134 and, where evidence does exist, it agrees less often than that for ecological outcomes, resulting

135 in low confidence (Figure 4). For ecological outcomes, 25\% exhibited high quantity and quality

136 evidence and $44 \%$ showed a high level of agreement among evidence (see data provided with

137 Supplementary Information). In contrast, no social or economic outcome exhibited high

138 quantity and quality evidence and only $21 \%$ showed a high level of agreement among evidence.

139 Further, where high confidence was determined for social and economic outcomes, the effect

140 direction on outcomes across ABMTs was not always consistent. While we found high

141 confidence that some ABMTs have positive effects on increasing non-harvest earnings,

142 alternative livelihood activities, maintaining access to resources, and preserving traditions,

143 others were found to have no effect or negative effects on the same outcome. The direction of

144 effects on social and economic outcomes were generally less consistent across tools than was

145 found for ecological outcomes.

147 Linking ABMTs to SDG 14 targets

148 We found that the assessed ABMTs could potentially contribute to five of the seven SDG 14 149 targets (Figure 5): SDG 14.2 (marine ecosystems), SDG 14.4 (sustainable fisheries), SDG 14.5

150 (conservation), SDG 14.6 (harmful subsidies), and SDG 14.7 (Small Island Developing States)

151 based on qualitatively determined ABMT scores (see Methods). Since we determined with high

152 confidence that most or all of the assessed ABMTs have no effect on reducing pollution or the

153 impacts of ocean acidification (Figure 4), we found no strong evidence that these tools

154 contribute to SDG 14.1 (marine pollution) or SDG 14.3 (ocean acidification). Based on the 155 confidence assessments presented here, there is currently no evidence that PSSAs on their own 156 contribute to SDG 14 targets. We found that multi-sector ABMTs (i.e., FPAs, PPAs, and LMMAs) 157 make greater potential contributions to targets that focus on ecological objectives, including 
SDG 14.2, 14.4, and 14.5, than single-sector ABMTs (i.e., GRAs, FCs, and TURFs). This reflects the high confidence identified for positive effects of multi-sector ABMTs on more ecological outcomes than single-sector ABMTs (Figure 4). For all ABMTs, except PSSAs, the potential contributions made to these targets were greater than potential contributions to targets with social and economic objectives, including SDG 14.6 and 14.7.

164 While multi-sector ABMTs might make similar contributions to ecologically focused SDG 14 targets, they differ in their potential to contribute to socially and economically focused targets

166 (Figure 5). FPAs emerged as the tool with the greatest potential to contribute to SDGs 14.2,

167 14.4, and 14.5; however, this tool had lower ABMT scores than LMMAs for contributing to SDG

16814.6 and 14.7. PPAs had the lowest ABMT scores for contributing to SDG 14.6 and 14.7 of all assessed ABMTs, with the exception of FCs and PSSAs, which were not found to make

170 contributions to these targets based on current evidence. We identified LMMAs as the ABMT

171 with the greatest potential to contribute to SDG 14.6 and 14.7. Second to LMMAs, TURFS

172 presented higher ABMT scores for these targets than other tools. Both LMMAs and TURFs were

173 the only tools found to increase harvest earnings and maintain access to resources with high

174 confidence (Figure 4), thus supporting their greater potential to contribute to these targets. FCs

175 were the only single-sector ABMT found to make no contribution to SDG 14.6 and 14.7. This is

176 likely due to the negative effect of FCs on maintaining access to resources (Figure 4), an

177 outcome that supports these targets (see data provided with Supplementary Information).

\section{DISCUSSION}

180 We found that, based on current evidence, FPAs hold the greatest potential to contribute to 181 ecologically focused SDG 14 targets (i.e., SDG 14.2, 14.4, and 14.5), aiming to preserve or 182 restore ocean ecosystems and biodiversity, followed by PPAs and LMMAs (Figure 5). Those 183 three tools typically regulate multiple sectors and activities, while the remaining tools target 184 activities of single sectors, particularly fisheries via GRAs, FCs, and TURFS and shipping activities 185 via PSSAs. Our results indicate that these multi-sector ABMTs, which may involve the use of 186 multiple single-sector ABMTs, will be important for achieving SDG 14, given their strong 
187 potential to contribute to multiple targets. Since no single ABMT assessed was found to 188 potentially contribute to all targets, nor was a single ABMT identified as holding the greatest 189 potential to contribute to both ecologically and socially and economically focused targets, our 190 findings highlight the need for a combination of tools to meet SDG 14. This resonates with a 191 preliminary analysis by the United Nations of spatial management strategies, including marine 192 spatial planning and integrated coastal zone management, for achieving ocean-related SDGs ${ }^{6}$.

193 Our results also highlight the potential role of these ABMTs in contributing to conservation 194 targets as OECMs. While we determined similar ABMT scores for multi-sector tools, an 195 important distinction between FPAs, PPAs, and LMMAs lies in their overarching objectives: 196 biodiversity conservation is the key goal of FPAs and PPAs ${ }^{21}$, while LMMAs prioritize sustainable 197 resource use over conservation per $s^{22}$.

199 LMMAs, a tool that has been favoured in Small Island Developing States ${ }^{23}$, are unique in their 200 application of a suite of ABMTs under a shared management strategy that is collaborative 201 across communities, partner organizations, and governments at the local level ${ }^{24}$. These 202 characteristics are likely reflected in the ABMT scores we present here (Figure 5), particularly 203 due to their ability to deliver ecological, social, and economic outcomes with high confidence 204 (Figure 4). Despite the relatively limited evidence of LMMA outcomes in the primary literature 205 (Table 1), this ABMT scored similarly to FPAs and PPAs that are well-documented. It is clear 206 from our results that experts agree on generally positive expected effects of LMMAs on 207 outcomes (Figure 3). Given these findings, LMMAs represent an opportunity for management 208 as a potential pathway and a research priority to support the achievement of SDG 14. As a tool 209 for ensuring a healthy and resilient ocean for sustainability ${ }^{25,26}$, FPAs undoubtedly have a 210 central role in achieving SDG 14.2, 14.4, and 14.5; however, the overarching aim of LMMAs may 211 enable greater delivery of outcomes contributing to SDG 14 targets that seek to sustain the 212 social and economic systems affecting oceans and ocean resources.

214 While our results show how the assessed ABMTs can potentially contribute SDG 14 targets, 215 they also highlight limitations of ABMTs. We found "incomplete" evidence for all assessed 
216 outcomes of PSSAs (Figure 4), hence, no potential for PSSAs to contribute to SDG 14 (Figure 5).

217 PSSAs may make other important contributions to conservation and sustainability by reducing

218 impacts from international shipping activities. This tool has been suggested for use in areas

219 beyond national jurisdiction for conservation 27,28 , where threats to biodiversity are growing ${ }^{29,30}$.

220 We highlight a need for more research on PSSA outcomes to support this tool as a potential

221 pathway to SDG 14 in the high seas and suggest that ABMTs contributing to conservation

222 outcomes should be used until the role of PSSAs can be clarified, such as FPAs. Our results also

223 demonstrate the inability of the assessed ABMTs to effectively reduce marine pollution (SDG

224 14.1) and impacts of ocean acidification (SDG 14.3) (Figure 5). Other ABMTs not assessed here,

225 such Special Areas designed under the MARPOL Convention ${ }^{31}$, may better support these

226 targets. Our results may reflect the broader and more systemic changes required for addressing

227 these issues; for example, regulating the consumption and disposal of plastic or large-scale

228 actions for reducing carbon emissions and decarbonizing economies ${ }^{32-34}$. Such systemic

229 transformations may be necessary precursors to effectively achieving SDG 14.1 and 14.3, for

230 which little progress has been made ${ }^{35}$. It will be important to consider both non-spatial and

231 spatial management tools, including and beyond the ABMTs assessed here, to achieve all SDG

23214 targets. Holistic approaches to planning and management across the land-sea interface, like

233 integrated coastal zone management ${ }^{36}$ and ridge-to-reef management ${ }^{37}$, will likely be

234 important for incorporating land-based regulations with ABMTs to achieve SDGs, including SDG

$23514^{6}$.

236

237 Through this work, we have identified several evidence gaps pertaining to ABMTs and their

238 outcomes. We highlight low confidence and a lack of evidence pertaining to the social and

239 economic outcomes of ABMTs (Table 1, Figure 3, Figure 4). Low confidence was found for $61 \%$

240 of social and economic outcomes assessed, compared to only $25 \%$ of ecological outcomes.

241 These findings indicate a need for research to better assess social and economic outcomes of

242 ABMTs at varying scales and for diverse stakeholders, which may first require the development

243 of measurable indicators for targets that are presently without ${ }^{38}$. This evidence gap is again

244 highlighted by the ABMT scores presented here, which indicate the low potential of these tools 
245 to contribute to SDG 14.6 and 14.7 that are socially and economically focused. While recent

246 work helped to identify social and economic outcomes of marine protected areas ${ }^{39}$, an

247 apparent evidence gap persists in identifying these outcomes for single-sector ABMTs, including 248 FCs, GRAs, TURFs, and PSSAs.

250 We recognize the inherent limitations of our study imposed by the rapid systematic review

251 method. While efforts were made to ensure that this method captured all relevant information,

252 it cannot be truly comprehensive as would a full systematic review capturing both primary and

253 grey literature. Our focus on primary, peer-reviewed literature provided a common filter,

254 ensuring a consistent rigour across the collected evidence. This method was used to conduct a

255 high-level confidence assessment that relied on a qualitative ranking system to assess the

256 quantity and quality of evidence (Supplementary Table 6). To compensate for differences in the

257 availability of literature-based evidence, we surveyed experts to identify the expected effects of

258 ABMTs. Through the selection of survey participants in primarily research institutions and

259 government, expertise from industry, Indigenous Peoples, and communities are not present in

260 our confidence assessments. As with any scientific synthesis, our work may be affected by

261 publication bias with some ABMTs more represented in the literature than others, as well as a

262 tendency for literature to report positive outcomes. As more evidence becomes available, the

263 confidence assessments and potential contributions of tools to SDG 14 targets presented here

264 may be refined. Our results demonstrate the qualitative potential of ABMTs to contribute to

265 SDG 14 and their use in practice should appropriately reflect this. Further, while we did select

266 prominent ABMTs for assessment, other tools are available to practitioners and may also prove

267 useful for achieving SDG 14. Our study does not assess the efficacy of ABMTs, nor does it

268 quantify the extent of outcomes. While ABMTs may deliver similar outcomes, one tool may

269 have a stronger effect than others and our study does not account for this. Delivery of these

270 outcomes is dependent on local social-ecological systems and may vary by target species,

271 ecosystems, and management regimes. To reach their fullest potential, ABMTs must be

272 appropriately designed with attention given to local needs, actively managed, and well-

273 enforced to consistently deliver outcomes contributing to SDG $14^{40-42}$. 
275 ABMTs are one type of management tool in a suite of tools and approaches available to

276 decision-makers for achieving conservation and sustainability objectives. Our results confirm

277 that no single ABMT can be used to reach all SDG 14 targets, but that a combination of tools,

278 especially those regulating multiple sectors, will likely be necessary for achieving this goal.

279 When multiple ABMTs are used synergistically, multiple and more diverse outcomes may occur,

280 potentially making stronger contributions to SDG 14. Placing ABMTs into the wider picture of

281 integrated land-sea management, including both spatial and non-spatial approaches, will be

282 important for achieving ocean conservation and sustainability goals ${ }^{43,44}$. This is especially true

283 for SDG 14 targets that may not be met using ABMTs, including SDG 14.1 to reduce marine

284 pollution and SDG 14.3 to reduce ocean acidification as our results indicate. Our findings

285 highlight important evidence gaps related to social and economic outcomes of ABMTs,

286 especially single-sector ABMTs, providing a research agenda for future work. Through our

287 assessment, we demonstrate which ABMTs may be most useful for achieving specific SDG 14

288 targets, allowing ocean planners and practitioners to make strategic decisions when selecting

289 management tools. By linking ABMTs to SDG 14 targets, our work may support future research

290 to assess potential contributions of existing or planned ABMTs to SDG 14 at a regional, national,

291 and international scale.

\section{METHODS}

\section{Selecting ABMTs and outcomes}

295 The ABMTs assessed in this study were identified from a list of spatial management tools 296 provided by Ehler and Douvere ${ }^{10}$, which included marine protected areas (MPAs), fishery 297 closures (FCs), gear restriction areas (GRAs), and particularly sensitive sea areas (PSSAs). Here, 298 we separated the broad category of MPAs into two distinct tools: fully protected areas (FPAs) 299 and partially protected areas (PPAs) due to their documented differences in outcomes ${ }^{45}$. Locally 300 managed marine areas (LMMAs) and territorial user right fisheries (TURFs) were added, as they 301 have emerged prominently in the literature in recent years ${ }^{46,47}$. In total, we identified seven 302 ABMTs with distinct objectives, regulations, and outcomes, though these definitions may vary 
or overlap in their application (Figure 1 and Supplementary Table 2). Some ABMTs may overlay tools, for example an ABMT regulating multiple sectors, like an FPA, may make use of multiple single-sector tools, like FCs, GRAs, or PSSAs.

We assessed 17 ecological, social, and economic outcomes identified from previous reviews reporting observed and expected ABMT outcomes, particularly in the context of MPAs ${ }^{48}$ and LMMAs $^{11}$ (Figure 1). Outcomes were assigned distinct definitions (Supplementary Table 1), and evidence of outcomes reported in literature needed to fit these definitions to be included in review. Some of these outcomes have direct links to SDG 14 targets (e.g., maintaining

312 ecosystem resilience and SDG 14.2 on ecosystem restoration), while others are indirect (e.g., 313 maintaining equitable access to resources and SDG 14.4 on sustainable fisheries). Since the 314 primary aim of this research is to provide guidance on the use of ABMTs to achieve SDG 14 315 targets, additional outcomes not previously identified from existing reviews but with direct links 316 to targets, such as reducing pollution and SDG 14.1, were added. Many of the assessed 317 outcomes may be considered as indicators for monitoring and evaluation of ABMTs ${ }^{49-51}$, which 318 may inform or align with SDG 14 indicators.

\section{Rapid systematic review}

321 Rapid systematic reviews identify all studies meeting specific criteria, such as publication year, 322 article type, geographic region, language, database, data type, or data extraction method ${ }^{52}$. We 323 used a rapid review method to overcome challenges introduced by the number of ABMTs and 324 outcomes assessed and the vastness of literature in this field of study. The review was 325 conducted using the Web of Science between July and October 2019, and was limited to 326 primary literature, including only articles and reviews published in English in 2002 or later.

327 While the grey literature could have provided additional information relevant to this study, 328 including this information would not have permitted a systematic review due to the diversity of 329 access restriction and languages that can be found in the grey literature. Since this method 330 excludes publications from governments, non-governmental organizations, and other research 331 organizations, we also conducted an expert opinion survey to compensate for evidence not 
332 found in the primary literature. The constraint on publication year was used to identify studies

333 listing author email addresses, which were later used as contact information to invite

334 participants for this survey ${ }^{53}$. We conducted two phases of rapid systematic review.

336 The first phase of rapid review was intended to capture synthesis-based evidence, including

337 meta-analyses, systematic reviews, and standard literature reviews, and thus it was assumed

338 that publications earlier than 2002 would have been included in these studies. Where

339 synthesis-based evidence was identified, further review of individual studies in the second

340 phase was not required, reducing the risk of double counting outcomes from individual studies

341 that may have been captured by reviews. In addition to these limitations, search terms were

342 designed to return the most relevant literature from titles, abstracts, and keywords

343 (Supplementary Tables 3 and 4). The efficacy of search terms was tested using pilot searches to

344 ensure that 10 pre-determined studies were returned by the selected search terms in Web of

345 Science (Supplementary Table 5).

347 Search terms were designed for the seven ABMTs, for ABMT outcomes in general, for meta348 analyses and reviews, and for the 17 outcomes assessed in this study. All returned literature 349 was screened first by reviewing titles and abstracts and then by reviewing full articles where an 350 inclusion decision could not be made confidently from the title and abstract. Literature was 351 screened based on the following criteria:

352 - Population: studies must observe the marine environment

353 - Intervention: studies must observe at least one of the seven ABMTs, aligning with the 354 assigned definitions (Supplementary Table 2), though evidence of any assessed ABMT $355 \quad$ was recorded

356 - Time and place: studies must be published after 2001 in any geographic location

357 - Outcomes: studies must report evidence of positive, negative, or no effect on at least 358 one of the 17 selected outcomes (Supplementary Table 1), excluding studies using 359 strictly theoretical methods or theoretical modelling 
361 In the second phase of the review, additional searches were conducted to identify studies

362 reporting evidence of individual ABMT outcomes for any case where synthesis-based evidence

363 was not identified in the first phase of review. Returned literature for each outcome per ABMT

364 was sorted using the "Relevance" feature on Web of Science, which ranks studies based on

365 search term frequency in titles, abstracts, and keywords. Then, the first 25 records were

366 screened using the same method and inclusion criteria as the first review phase. In cases where

367 fewer than 10 records met all inclusion criteria, the next 25 records available in Web of Science

368 were screened. We used this method to ensure that the most relevant literature and best

369 available evidence was captured.

370

371 In the first phase, when studies met all inclusion criteria, the following data were extracted:

372 study type (i.e., meta-analysis, systematic review, or standard literature review), number of

373 studies reviewed or included in the meta-analysis, geographical scope, ABMT type, outcome

374 type (i.e., ecological, social, economic, or multiple), evidence of outcomes, and effect directions

375 (e.g., positive effect on organism size). Additional information related to study quality,

376 including the reporting and rigour of methods, was recorded and informed distinctions made

377 between study type where necessary. Systematic literature reviews were distinguished from

378 standard literature reviews such that the former reported a search strategy, including search

379 terms, and inclusion/exclusion criteria. For each study included, the reported ABMT was

380 classified according to the definitions used in this study (Supplementary Table 2), which in some 381 cases differed from the ABMT that was searched in Web of Science. For example, if a study was

382 returned by a search for FC outcomes, but the described ABMT fit this study's definition of a 383 GRA, the ABMT was classified as a GRA. Where it was unclear whether an MPA was fully or 384 partially protected, MPAtlas (http://www.mpatlas.org/) was used to determine the appropriate 385 ABMT type. If an MPA was reported as being entirely no-take on MPAtlas, it was classified as an 386 FPA. All other MPAs, including those with some no-take zones according to MPAtlas, were 387 classified as PPAs. In the second phase, data on study location, ABMT type, evidence of 388 outcomes, and effect direction were extracted and qualitative information that clarified the 389 nature of reported outcomes was recorded. 


\section{Expert opinion survey}

391 An expert opinion survey was designed to capture knowledge of ABMT outcomes that could fill

392 gaps from the rapid systematic review. Using Qualtrics ${ }^{\circledR}$ software, the online survey asked

393 participants to identify their familiarity with the assessed ABMTs, the expected effect of ABMTs

394 on each outcome, and to provide demographic information relating to their professional

395 experience. When identifying the expected effects of ABMTs on outcomes, participants were

396 asked to assume that ABMTs were appropriately designed, actively managed, and well-

397 enforced. We identified potential participants using two methods: (1) authors of studies

398 included in the first phase of review and (2) known experts in the ABMTs identified from our

399 collective professional networks, including representatives of academia, governments, non-

400 governmental organizations, and independent experts. Invitations to participate were extended

401 to these individuals via direct email with an anonymous link to the survey. In addition, we

402 invited participants to share the survey with colleagues or to provide contact information of

403 colleagues to receive an individual invitation. To conduct the confidence assessment, responses

404 on the expected effects of ABMTs were grouped into positive effects from "Strong Positive" and

405 "Positive" responses, negative effects from "Strong Negative" and "Negative" responses, and

406 uncertain effects from "Do Not Know" and "Prefer Not to Answer" responses.

408 This research was approved by the Interdisciplinary Committee on Ethics in Human Research

409 (ICEHR) at the Memorial University of Newfoundland, ICEHR No. 20200294-AR.

\section{Confidence assessment}

412 To determine the level of confidence in the delivery of ABMT outcomes, we used an

413 assessment method modified from the IPBES assessment process ${ }^{20}$ (Figure 2, Supplementary

414 Table 6, Supplementary Figure 3). We defined five confidence categories based on the quantity

415 and quality of evidence and level of agreement among evidence collected via rapid systematic

416 review and expert opinion survey. These categories are:

417 - Well-established: comprehensive evidence exists, and conclusions agree 
- Established but incomplete: general agreement among evidence, although limited evidence exists

- Established but unresolved: moderate evidence exists, although conclusions do not consistently agree or disagree

- Unresolved: comprehensive evidence exists, and conclusions do not agree

- Incomplete: limited evidence exists, recognizing major knowledge gaps

425 We used a qualitative ranking system to determine the quantity and quality of evidence and the 426 level of agreement to be either "high", "medium", or "low" based on the criteria presented in

427 Supplementary Table 6, applied in a decision tree presented in Supplementary Figure 3. High 428 quantity and quality evidence included one or more meta-analysis or systematic review or more 429 than one standard literature review or five or more individual studies and more than $75 \%$ of 430 experts reporting one expected effect. High agreement required the majority of studies to 431 agree on an ABMT outcome, the majority of experts to agree on an outcome, and for the 432 studies and experts to agree on that same outcome. Each ABMT outcome was assessed 433 according to these criteria and assigned a confidence category (Supplementary Figure 3). Well434 established and established but incomplete categories were considered to be of high 435 confidence, reflecting high certainty that a particular ABMT would deliver a particular outcome, 436 and were assigned confidence scores of 2 and 1, respectively. All other confidence categories 437 were not assigned a confidence score, as these categories were considered to be of low 438 confidence. Linking ABMTs to SDG 14 targets

441 ABMT outcomes assessed in this study do not all make equivalent contributions to SDG 14 442 targets. To account for this, each outcome was assigned a contribution score per target. Scores 443 ranged from one to three, depending on the type of contribution. A direct contribution (score=

4443 ) has a central role in meeting the objectives of the target; a supporting contribution (score $=2$ ) 445 aids the delivery of a direct contribution; and an indirect contribution (score $=1$ ) enables the 446 delivery of supporting or direct contributions to a lesser extent (see data provided with 
447 Supplementary Information). We considered contributions to the targets broadly based on their 448 description and, where feasible, their indicators. While some indicators were used to inform 449 contribution scores for ABMT outcomes, others were not relevant to the outcomes assessed. 450 For example, a study reporting an ABMT outcome of healthy ocean acidity (pH), the indicator 451 for SDG 14.3, would be considered as evidence of a direct contribution to this target. Similarly, 452 a study reporting improved ecosystem resilience as an ABMT outcome would be considered as 453 evidence of an indirect contribution to this target (see data provided with Supplementary 454 Information). In contrast, the indicator for SDG 14.5, protected area coverage, is not an 455 outcome of ABMTs and is therefore not relevant to this study. To link ABMTs to SDG 14 targets, 456 an ABMT score was calculated according to the following equation:

$$
A B M T \text { score }=\Sigma \text { (Confidence score } \times \text { Contribution score) }
$$

458 Possible ABMT scores ranged between 0 and 102, depending on the assigned confidence and 459 contribution scores for the 17 assessed outcomes (see data provided with Supplementary 460 Information). ABMT scores were qualitatively compared to determine the relative contributions 461 of ABMTs to SDG 14 targets (Supplementary Figure 4).

\section{DATA AVAILABILITY}

464 Data supporting the analyses and results of this study are available in the Supplementary

465 Information. Correspondence regarding this data should be addressed to J.M.R.

\section{REFERENCES}

468 1. United Nations. Transforming our world: the 2030 Agenda for Sustainable Development. 469 Gen. Assem. 70 Sess. 16301, 1-35 (2015).

470 2. Singh, G. G. et al. A rapid assessment of co-benefits and trade-offs among Sustainable 471 Development Goals. Mar. Policy 93, 223-231 (2018).

472 3. Claudet, J. et al. Perspective A Roadmap for Using the UN Decade of Ocean Science for 473 Sustainable Development in Support of Science, Policy, and Action. One Earth 2, (2020).

474 4. Ntona, M., Morgera, E. \& Ntona, M. Connecting SDG 14 with the other Sustainable 475 Development Goals through marine spatial planning. Mar. Policy 1-9 (2017). 
doi:10.1016/j.marpol.2017.06.020

477 5. Cormier, R. \& Elliott, M. SMART marine goals, targets and management - Is SDG 14 operational or aspirational, is 'Life Below Water' sinking or swimming? Mar. Pollut. Bull. 123, 28-33 (2017).

6. United Nations Environment Programme. Preliminary analysis of area-based management measures to support SDG implementation. (2017).

7. Diz, D. et al. Mainstreaming marine biodiversity into the SDGs: The role of other effective area-based conservation measures (SDG 14.5). Mar. Policy 93, 251-261 (2018).

8. Haas, B., Fleming, A., Haward, M. \& McGee, J. Big fishing: the role of the large-scale commercial fishing industry in achieving Sustainable Development Goal 14. Rev. Fish Biol. Fish. 8, 161-175 (2019).

9. Claudet, J., Roussel, S. \& Pelletier, D. Spatial management of near shore coastal areas: the use of Marine Protected Areas (MPAS) in a fisheries management context. Vie MilieuLife Environ. 56, 301-305 (2006).

10. Ehler, C. \& Douvere, F. Marine Spatial Planning: a step-by-step approach toward ecosystem-based management. Intergov. Oceanogr. Comm. Man Biosph. Program. IOC Manual, 1-98 (2009).

11. Jupiter, S. D., Cohen, P. J., Weeks, R., Tawake, A. \& Govan, H. Locally-managed marine areas: Multiple objectives and diverse strategies. Pacific Conserv. Biol. 20, 165-179 (2014).

13. Palmer, M. C. \& Demarest, C. Getting to good enough: Performance of a suite of methods for spatially allocating fishing effort to management areas. Fish. Res. 204, 26-32

501 14. Agardy, T., di Sciara, G. N. \& Christie, P. Mind the gap: Addressing the shortcomings of marine protected areas through large scale marine spatial planning. Mar. Policy 35, 226503232 (2011).

504 15. Frazão Santos, C. et al. Integrating climate change in ocean planning. Nat. Sustain. 
(2020). doi:10.1038/s41893-020-0513-x

506 16. Molenaar, E. J. Area-based management tools. (2013).

507 17. Johnson, D., Adelaide Ferreira, M. \& Kenchington, E. Climate change is likely to severely

508 limit the effectiveness of deep-sea ABMTs in the North Atlantic. Mar. Policy 87, 111-122 $509 \quad$ (2018).

510 18. Secretariat of the Convention on Biological Diversity, Food and Agriculture Organisation 511 of the United Nations, The World Bank, United Nations Environment Programme \& 512 United Nations Development Programme. Biodiversity and the 2030 Agenda for $513 \quad$ Sustainable Development Biodiversity Is Essential for Sustainable Development. (2016).

514 19. De Santo, E. M. Implementation challenges of area-based management tools (ABMTs) for 515 biodiversity beyond national jurisdiction (BBNJ). Mar. Policy 97, 34-43 (2018).

$51620 . \quad$ Intergovernmental Panel on Biodiversity and Ecosystem Services. The IPBES Assessment $517 \quad$ Process. (2016).

518 21. Sciberras, M. et al. Evaluating the relative conservation value of fully and partially 519 protected marine areas. Fish Fish. 16, 58-77 (2015).

520 22. Burke, L., Reytar, K., Spalding, M. \& Perry, A. Reefs at Risk Revisited. (World Resources $521 \quad$ Institute, 2011).

522 23. Island Voices Global Choices. In-depth analysis of partnerships for Small Island 523 Developing States. (2018).

524 24. Govan, H. Status and potential of locally-managed marine areas in the Pacific Island Region: meeting nature conservation and sustainable livelihood targets through widespread implementation of LMMAs. (2009). doi:SPREP/WWF/WorldFish-Reefbase/CRISP

527 25. Giakoumi, S. et al. Ecological effects of full and partial protection in the crowded 528 Mediterranean Sea: a regional meta-analysis. Sci. Rep. 7, 8940 (2017).

529 26. Organization for Economic Co-operation and Development. Marine Protected Areas: 530 Economics, Management and Effective Policy Mixese. (OECD Publishing, 2017). 531 doi:https://doi.org/10.1787/9789264276208-en.

532 27. Rochette, J. et al. Delivering the Aichi target 11: Challenges and opportunities for marine 533 areas beyond national jurisdiction. Aquat. Conserv. Mar. Freshw. Ecosyst. 24, 31-43 
(2014).

28. Roberts, J., Chircop, A. \& Prior, S. Area-based Management on the High Seas: Possible Application of the IMO’s Particularly Sensitive Sea Area Concept. Int. J. Mar. Coast. Law 25, 483-522 (2010).

29. Warner, R. M. Conserving marine biodiversity in areas beyond national jurisdiction: Coevolution and interaction with the law of the sea. Front. Mar. Sci. 1, 1-11 (2014).

30. Gjerde, K. M. et al. Protecting Earth's last conservation frontier: scientific, management and legal priorities for MPAs beyond national boundaries. Aquat. Conserv. Mar. Freshw. Ecosyst. 26, 45-60 (2016).

31. International Maritime Organization. Special Areas Under MARPOL. (2020).

32. Stafford, R. \& Jones, P. J. S. Viewpoint - Ocean plastic pollution: A convenient but distracting truth? Mar. Policy 103, 187-191 (2019).

33. Blythe, J. et al. The Dark Side of Transformation: Latent Risks in Contemporary Sustainability Discourse. Antipode 0, 1-18 (2018).

34. Geels, F. W., Sovacool, B., Schwanen, T. \& Sorrell, S. Sociotechnical transitions for deep decarbonization: Accelerating innovation is as important as climate policy. Science (80-. ). 357, 1242-1244 (2017).

35. United Nations. The sustainable development goals report 2019. United Nations publication issued by the Department of Economic and Social Affairs (2019).

36. Pittman, J. \& Armitage, D. Governance across the land-sea interface: A systematic review. Environ. Sci. Policy 64, 9-17 (2016).

37. Delevaux, J. M. S. et al. A linked land-sea modeling framework to inform ridge-to-reef management in high oceanic islands. PLOS ONE 13, (2018).

38. Recuero Virto, L. A preliminary assessment of the indicators for Sustainable Development Goal (SDG) 14 "Conserve and sustainably use the oceans, seas and marine resources for sustainable development". Mar. Policy 98, 47-57 (2018).

39. Ban, N. C. et al. Well-being outcomes of marine protected areas. Nat. Sustain. 2, 524-532 (2019).

40. Bennett, N. J. \& Dearden, P. Why local people do not support conservation: Community 
perceptions of marine protected area livelihood impacts, governance and management in Thailand. Mar. Policy 44, 107-116 (2014).

565 41. Devillers, R. et al. Reinventing residual reserves in the sea: Are we favouring ease of establishment over need for protection? Aquat. Conserv. Mar. Freshw. Ecosyst. 25, 480504 (2015).

42. Gill, D. A. et al. Capacity shortfalls hinder the performance of marine protected areas globally. Nature 543, 665-669 (2017).

43. Álvarez-Romero, J. G. et al. Integrated cross-realm planning: A decision-makers' perspective. Biol. Conserv. 191, 799-808 (2015).

572 44. Reuter, K. E., Juhn, D. \& Grantham, H. S. Integrated land-sea management: Recommendations for planning, implementation and management. Environ. Conserv. 43, 181-198 (2016).

575 45. Zupan, M. et al. Marine partially protected areas: drivers of ecological effectiveness. Front. Ecol. Environ. 16, 381-387 (2018).

577 46. Rocliffe, S., Peabody, S., Samoilys, M. \& Hawkins, J. P. Towards a network of locally managed marine areas (LMMAs) in the Western Indian Ocean. PLoS One 9, e103000e103000 (2014).

47. Lester, S. E., McDonald, G., Clemence, M., Dougherty, D. T. \& Szuwalski, C. S. Impacts of TURFs and marine reserves on fisheries and conservation goals: Theory, empirical evidence, and modeling. Bull. Mar. Sci. 93, 173-198 (2017).

48. Bennett, N. J. \& Dearden, P. From measuring outcomes to providing inputs: Governance, management, and local development for more effective marine protected areas. Mar. Policy 50, 96-110 (2014).

588 50. Kincaid, K., Rose, G. \& Devillers, R. How fisher-influenced marine closed areas contribute to ecosystem-based management: A review and performance indicator scorecard. Fish Fish. 18, 860-876 (2017).

591 51. Pomeroy, R. S., Parks, J. E. \& Watson, L. M. How Is Your MPA Doing? A Guidebook of 
594 52. Ganann, R., Ciliska, D. \& Thomas, H. Expediting systematic reviews: Methods and 595 implications of rapid reviews. Implement. Sci. 5, 1-10 (2010).

596 53. Sinclair, S. P. et al. The use, and usefulness, of spatial conservation prioritizations.

597 Conserv. Lett. 11, 1-7 (2018).

599 CORRESPONDING AUTHOR

600 All correspondence regarding this manuscript and requests for materials can be directed to 601 J.M.R.

602

\section{ACKNOWLEDGEMENTS}

604 We thank Dr. Natalie Ban for her support in this work, including survey development, and 605 useful comments on an earlier version of this manuscript. This work was supported by the 606 Mitacs Globalink Research Award and a Discovery Grant of the Natural Sciences and 607 Engineering Research Council (NSERC) of Canada awarded to R.D. JC was supported by 608 BiodivERsA (METRODIVER) and Agence Nationale de la Recherche (ANR-14-CE03-0001-01).

\section{AUTHOR CONTRIBUTIONS}

611 All authors contributed to the conception of this research, to study design, and to editing the 612 manuscript. J.M.R. reviewed the literature, conducted the expert opinion survey, carried out 613 analyses, and drafted the manuscript.

\section{COMPETING INTERESTS}

616 The authors declare no competing interests. 
620 Figure 1. Area-based management tools and their potential ecological, social, and economic

621 outcomes assessed in this study. The selected tools have objectives for biodiversity

622 conservation, sustainable resource use, or both and regulate activities of single or multiple

623 sectors. Complete definitions of tools and outcomes are provided in Supplementary Tables 1

624 and 2. This figure presents positive effects on outcomes given their potential contributions to

625 SDG 14, though positive, negative, and neutral effects were collected as evidence in this study.

626 FPA, fully protected area; PPA, partially protected area; LMMA, locally managed marine area;

627 GRA, gear restriction area; FC, fishery closure; TURF, territorial user right fishery; PSSA,

628 particularly sensitive sea area. Icons are attributed to Becris (LMMA), Freepik (FPA, PPA),

629 Mavadee (PSSA), Smashicons (FC), Surang (GRA), Wichai.wi (TURF) from www.flaticon.com.

631 Figure 2. Confidence assessment framework, adapted from IPBES ${ }^{20}$, used to determine 632 confidence in the delivery of area-based management tool (ABMT) outcomes based on 633 evidence from rapid systematic review and expert opinions. Criteria for determining quantity 634 and quality of evidence and level of agreement among evidence are defined in Supplementary

635 Table 6 and detailed in Supplementary Figure 3. "Well-established" and "established but 636 incomplete" categories were considered to be high confidence categories, while remaining

637 categories were considered to be low confidence, for the purpose of linking ABMTs to 638 Sustainable Development Goal 14 targets (see Methods). Icons are attributed to Becris

639 (LMMA), Freepik (FPA, PPA), Mavadee (PSSA), Smashicons (FC), Surang (GRA), Wichai.wi (TURF) 640 from www.flaticon.com.

642 Figure 3. Expert opinions $(n=75)$ on the expected effects of area-based management tools 643 (ABMTs) on ecological, social, and economic outcomes. See Supplementary Tables 1 and 2 for 644 definitions of ABMTs and outcomes. FPA, fully protected area; PPA, partially protected area; 645 LMMA, locally managed marine area; GRA, gear restriction area; FC, fishery closure; TURF, 646 territorial user right fishery; PSSA, particularly sensitive sea area. 
648 Figure 4. Confidence assessment of literature-based and expert opinion evidence for the 649 delivery of ecological, social, and economic outcomes from area-based management tools 650 (ABMTs). There is highest confidence in the delivery of "well-established" outcomes (green) and 651 lowest confidence in the delivery of "incomplete" outcomes (dark orange). The effect direction

652 for each outcome is indicated within each circle. For some "established but unresolved" and 653 "unresolved" outcomes, more than one effect direction is indicated due to a lack of agreement 654 among evidence. "Incomplete" outcomes do not indicate an effect direction. FPA, fully 655 protected area; PPA, partially protected area; LMMA, locally managed marine area; GRA, gear 656 restriction area; FC, fishery closure; TURF, territorial user right fishery; PSSA, particularly 657 sensitive sea area. Icons are attributed to Becris (LMMA), Freepik (FPA, PPA), Mavadee (PSSA), 658 Smashicons (FC), Surang (GRA), Wichai.wi (TURF) from www.flaticon.com.

660 Figure 5. Potential contributions of area-based management tools (ABMTs) to Sustainable 661 Development Goal 14 (SDG 14) targets based on confidence assessments of ABMT outcomes 662 and the potential contributions of outcomes to SDG 14 targets (see data supplied with 663 Supplementary Information). Circle size is proportional to ABMT scores indicated in each circle 664 (scores range from 0 to 102, see Equation 1 in Methods). Crosses indicate an ABMT score of 665 zero, meaning no potential contribution of a tool to a target, based on current evidence and the 666 relative contribution of an outcome to a target. FPA, fully protected area; PPA, partially 667 protected area; LMMA, locally managed marine area; GRA, gear restriction area; FC, fishery 668 closure; TURF, territorial user right fishery; PSSA, particularly sensitive sea area. Icons are 669 attributed to Becris (LMMA), Freepik (FPA, PPA), Mavadee (PSSA), Smashicons (FC), Surang 670 (GRA), Wichai.wi (TURF) from www.flaticon.com.

\section{TABLES}

673

674 Table 1. Summary of literature-based evidence of area-based management tool (ABMT) 675 outcomes collected via rapid systematic review. Blue cells indicate outcomes from synthesis676 based evidence, including meta-analyses, systematic reviews, or literature reviews, and show 
677 the number of studies reporting evidence. White cells indicate the number of individual studies 678 reporting evidence, for any outcome that was not reported in synthesis-based evidence (see 679 Methods). Values represent one study per evidence record relating to an ABMT outcome, 680 though some studies reported evidence of more than one outcome or evidence from more 681 than one ABMT and are therefore counted in multiple cells where relevant. Evidence may 682 report positive (+), negative (-), or neutral effects (O) on the ABMT outcome. FPA, fully 683 protected area; PPA, partially protected area; LMMA, locally managed marine area; GRA, gear 684 restriction area; FC, fishery closure; TURF, territorial user right fishery; PSSA, particularly 685 sensitive sea area. 